Trauma Berufskrankh $2008 \cdot$ 10[Suppl 1]:64-68 DOI 10.1007/s10039-007-1259-7

Online publiziert: 17. Oktober 2007

๑) Springer Medizin Verlag 2007

\author{
S. Wicker ${ }^{1}$ H.F. Rabenau ${ }^{2}$ \\ ${ }^{1}$ Betriebsärztlicher Dienst, Klinikum der \\ Johann-Wolfgang-Goethe-Universität Frankfurt, Frankfurt am Main \\ ${ }^{2}$ Institut für Medizinische Virologie, Klinikum der \\ Johann-Wolfgang-Goethe-Universität Frankfurt, Frankfurt am Main
}

\title{
Nadelstichverletzungen im klinischen Alltag
}

\section{Ergebnisse der Frankfurter Nadelstichstudie}

Nadelstichverletzungen (NSV) bergen das Risiko einer Infektion mit

- dem Hepatitis-B-Virus (HBV),

- dem Hepatitis-C-Virus (HCV) oder

- dem humanen Immundefizienzvirus (HIV).

In Deutschland kommt es jedes Jahr zu schätzungsweise 500.00o NSV [4], diese verursachen Kosten von etwa 50 Mio. EUR [23]. NSV zählen zu den häufigsten Arbeitsunfällen der Mitarbeiter des Gesundheitswesens [3]. In diesen Kontext spielen Chirurgen bzw. D-Ärzte eine wichtige Rolle. Einerseits haben sie selbst aufgrund ihrer eigenen beruflichen Tätigkeit ein hohes Risiko, eine NSV zu erleiden $[16,20]$, andererseits stellen sie als DÄrzte in der Regel die ersten Ansprechpartner für betroffene Beschäftigte dar. In dieser Funktion erfüllen sie eine wichtige Lotsenfunktion, da sie den weiteren Behandlungsprozess maßgeblich beeinflussen. Eine Zeitverzögerung oder eine Fehlbehandlung an dieser Stelle können weit reichende Folgen nach sich ziehen [10].

Das Robert Koch-Institut (RKI) sowie die Deutsche und Österreichische AIDSGesellschaft empfehlen, dass in jedem Krankenhaus eine Handlungsanweisung gemäß der neuesten Richtlinien hinsichtlich der Behandlung von NSV zugänglich ist [2]. Jedes Krankenhaus sollte daher überprüfen, ob eine optimale Behandlung von NSV des Personals sichergestellt ist.

Weltweit infizieren sich aufgrund von NSV jedes Jahr etwa 66.00o Mitarbeiter des Gesundheitswesens mit HBV, schätzungsweise 16.000 mit $\mathrm{HCV}$ und etwa 1000 mit HIV [8].

Obwohl präventive Maßnahmen zur Vermeidung von NSV in den letzten Jahren verstärkt wurden, blieb deren Anzahl auf einem unbefriedigenden hohen $\mathrm{Ni}$ veau. Deswegen hat der Gesetzgeber in Deutschland mit der Technischen Regel für Biologische Arbeitsstoffe (TRBA 250) [7] den Einsatz von so genannten sicheren Instrumenten verbindlich geregelt. Diese reduzieren das Infektionsrisiko für Beschäftigte im Arbeits- und Gesundheitsdienst und verbessern den Schutz der Patienten $[6,11,12,15,21]$.

Vor dem Hintergrund der Neufassung der TRBA 250 (2006) wurde am Universitätsklinikum Frankfurt am Main vom Betriebsärztlichen Dienst und dem Institut für Medizinische Virologie eine Gefährdungsanalyse bezüglich NSV erstellt. Dabei wurde NSV definiert als

"Jegliche Stich-, Schnitt- und Kratzverletzung der Haut durch Kanülen, Skalpelle etc., die mit Patientenmaterial verunreinigt waren, unabhängig davon, ob die Wunde geblutet hat oder nicht - einschließlich des direkten Kontakts mit der Haut der Schleimhaut von Mund, Nase und Augen“.

Ziel der Studie waren die Erhebung der Anzahl sowie der Ursachen von $\mathrm{Na}$ delstichverletzungen und die Evaluation präventiver Maßnahmen.

\section{Methoden der Frankfurter Nadelstichstudie}

Das Universitätsklinikum Frankfurt am Main ist ein Klinikum der unabdingbaren Notfallversorgung mit 1247 Betten und 4080 Mitarbeitern [1365 Männer (33,5\%) und 2715 Frauen (66,5\%)]. Zwischen April und Juni 2006 führte der Betriebsärztliche Dienst in Zusammenarbeit mit dem Institut für Medizinische Virologie eine Fragebogenerhebung bezüglich Nadelstichverletzungen im Zentrum der Chirurgie, der Anästhesie, der Dermatologie, der Gynäkologie und der Kinder- und Jugendmedizin durch. In diesen Zentren sind insgesamt 919 Beschäftigte mit direktem $\mathrm{Pa}$ tientenkontakt bzw. mit Kontakt zu Untersuchungsmaterial eingesetzt. Es handelt sich hierbei um:

- $327(35,6 \%)$ Ärzte,

- $537(58,4 \%)$ Mitarbeiter aus dem Pflegedienst,

- 39 (4,3\%) Reinigungskräfte und

- 16 (1,7\%) Mitarbeiter in der Labordiagnostik.

Das ärztliche Personal sowie das Laborpersonal wurden im Rahmen der regelmäßig stattfindenden Besprechungen durch den Betriebsärztlichen Dienst über die Art der Erhebung in Kenntnis gesetzt. Das Pflege- und Reinigungspersonal wurden von den jeweiligen Gruppenleitungen über die Fragebogenerhebung informiert. 
Die Beschäftigten erhielten einen selbst entwickelten Fragebogen mit einer kurzen Einleitung über das Gefährdungspotenzial von Nadelstichverletzungen. Im Rahmen dieses Fragebogens wurden erfragt:

- die Anzahl der NSV innerhalb der letzten 12 Monate,

- die Art der invasiven Tätigkeit, bei der es zur NSV kam,

- das Arbeitsmittels, an dem sich der Mitarbeiter verletzte,

- das Meldeverhalten und

- die Berufsgruppe.

Der Fragebogen enthielt insgesamt 15 Items und 1 offene Frage bezüglich Anregungen und Anmerkungen zu NSV.

\section{Ergebnisse}

Von insgesamt 919 Mitarbeitern nahmen $720(78,3 \%)$ an der Studie teil. Der Fragebogenrücklauf der Ärzte lag bei 71,5\% $(\mathrm{n}=234 / 327)$, der des Pflegepersonals bei $75,4 \%(n=405 / 537)$. Darüber hinaus nahmen 81 Beschäftigte anderer Berufsgruppen (z. B. Reinigungskräfte, Laborangestellte) an der Erhebung teil. Der höchste Fragebogenrücklauf fand sich im Zentrum für Chirurgie, hier antworteten $82,2 \%(n=194 / 236)$ der Mitarbeiter auf die Befragung - davon 65,1\% $(\mathrm{n}=82 / 126)$ Ärzte und $92,7 \%(n=102 / 110)$ aus dem pflegerischen Bereich sowie 10 Mitarbeiter, die keine Angabe zur Berufsgruppe gemacht hatten.

Innerhalb der letzten 12 Monate hatten $31,4 \%$ aller Befragten $(n=226 / 720)$ mindestens eine NSV erlitten. Die Mitarbeiter aus dem Zentrum für Chirurgie wiesen mit 46,9\% die höchste Rate von NSV auf, wohingegen die Mitarbeiter der Kinderklinik mit $18,7 \%$ die am wenigsten betroffene Fachrichtung darstellten. Die Häufigkeit von NSV in den unterschiedlichen Fachbereichen ist in $\bullet$ Abb. 1 dargestellt.

Insgesamt 561 NSV wurden im Rahmen der anonymen Fragebogenerhebung angegeben (Spannweite: 1-55 NSV/12 Monaten). Während die betroffenen Mitarbeiter der konservativen Fächer in der Regel nur eine (bis maximal 2 NSV) in den letzten 12 Monaten aufwiesen, gaben die Mitarbeiter aus der Chirurgie bis zu

Trauma Berufskrankh 2008 · 10[Suppl 1]:64-68 DOI 10.1007/s10039-007-1259-7

c) Springer Medizin Verlag 2007

\section{S. Wicker · H.F. Rabenau \\ Nadelstichverletzungen im klinischen Alltag. Ergebnisse der Frankfurter Nadelstichstudie}

\section{Zusammenfassung}

Beschäftigte im Gesundheitswesen sind durch Nadelstich- und Schnittverletzung einer Infektionsgefährdung gegenüber durch Blut übertragbare Infektionen wie Hepatitis B (HBV), Hepatitis C (HCV) und HIV ausgesetzt. Ziel der Studie war die Erhebung der Häufigkeit und Ursachen von Nadelstichverletzungen (NSV) an einem deutschen Universitätsklinikum. Wir ermittelten den Anteil von NSV, die durch die Verwendung von sicheren Produkten hätten vermieden werden können: Innerhalb der letzten 12 Monate hatten $31,4 \%(n=226)$ der Befragten mindestens 1 NSV erlitten. Dabei war die NSV-Rate in der Chirurgie mit $46,9 \%$ besonders hoch. Durchschnittlich 36,4\% ( $n=204 / 561)$ der NSV hätten durch die Verwendung von sicheren Pro- dukten vermieden werden können. Dabei zeigte sich ein signifikanter Unterschied innerhalb der einzelnen Fachdisziplinen: Pädiatrie $82,6 \%$, Gynäkologie $81,4 \%$, Anästhesie $53,7 \%$, Dermatologie $41,7 \%$ und Chirurgie $14,6 \%$. Zahlreiche unabhängige Studien haben den Nutzen von sicheren Instrumenten belegt. Die Einführung sicherer Produkte wird zu einer Verbesserung des Arbeits- und Gesundheitsschutzes der medizinischen Beschäftigten führen und damit auch zu einem verbesserten Schutz der Patienten.

\section{Schlüsselwörter}

Beschäftigte in Gesundheitsberufen · Blutübertragbare Infektionen · Nadelstichverletzungen · Sichere Instrumente · TRBA 250

\section{Needlestick injuries in clinical routine. Results of the Frankfurt needlestick study}

\section{Abstract}

Healthcare workers (HCW) are exposed to bloodborne pathogens, especially hepatitis B (HBV), hepatitis C (HCV) and human immunodeficiency virus (HIV), through such job-related risk factors as needlestick, stab, scratch, and cut injuries or others that draw blood. The purpose of this study was to ascertain the frequency and the causes of needlestick injuries in a German university hospital. We calculated what proportion of the reported needlestick injuries could have been prevented by using safety devices. Within the previous 12 months $31.4 \%(n=226)$ of the HCWs taking part in the study had sustained at least one needlestick injury. HCWs in the department of surgery were most at risk, $46.9 \%$ of these having sustained needlestick injuries. On average $36.4 \%$ ( $n=204 / 561)$ of all needlestick injuries could have been avoid- ed by the use of safety devices. However, the various medical disciplines differ significantly in the proportion of needlestick injuries that could definitely have been avoided, this being highest in pediatrics $(82.6 \%)$, declining through gynecology $(81.4 \%)$, anesthesia $(53.7 \%)$, and dermatology (41.7\%) to surgery $(14.6 \%)$. Numerous independent studies have documented the benefit of using safety devices. The introduction of such instruments will lead to improved protection of the medical staff and through this in turn to better protection of the patients.

\section{Keywords}

Health care workers · Bloodborne viruses . Needlestick injuries · Safety devices . Technical Rule 250 

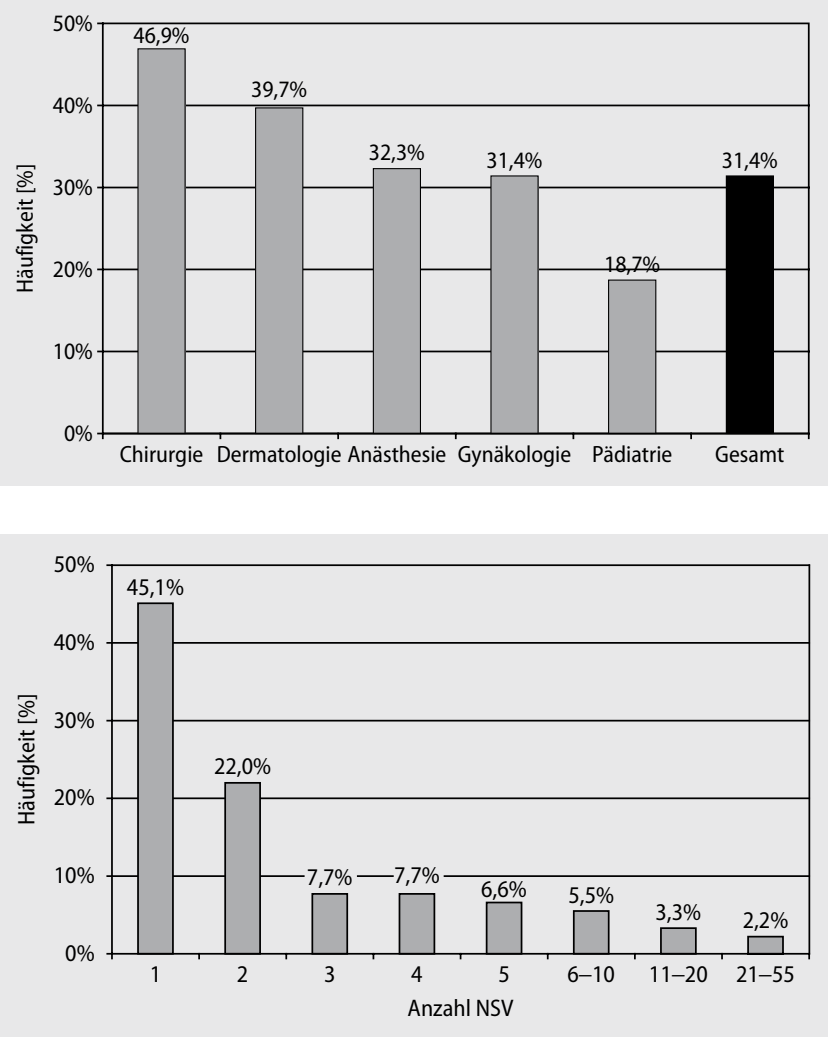

Abb. $1 \varangle$ Anteil der Mitarbeiter der einzelnen Fachdisziplinen mit NSV

Abb. $2 \triangleleft$ Anzahl von NSV innerhalb der letzten 12 Monaten bei betroffenen Mitarbeitern aus dem Zentrum für Chirurgie

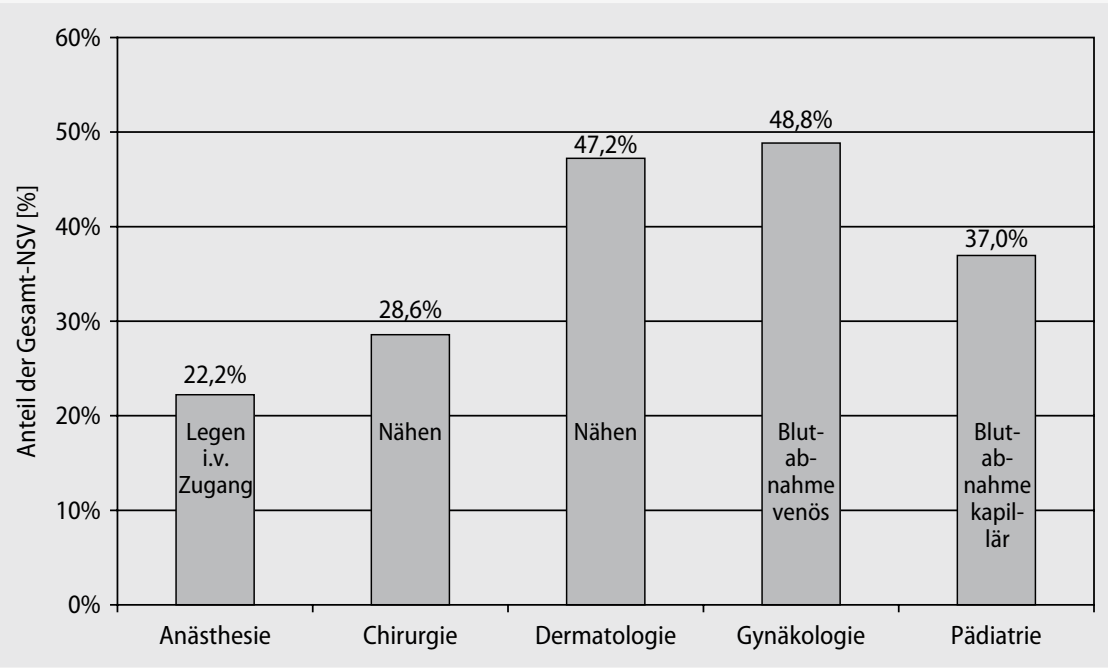

Abb. $3 \Delta$ Unfallschwerpunkte in den unterschiedlichen Fachdisziplinen

$55 \mathrm{NSV}$ in den vergangen 12 Monaten an (• Abb. 2).

Betrachtet man die einzelnen Berufsgruppen, hatten Ärzte insgesamt das höchste Risiko einer NSV. Insgesamt $55,1 \%$ der befragten Ärzte $(n=129 / 234)$ gaben mindestens 1 NSV innerhalb der letzten 12 Monate an, gefolgt von den Mitarbeitern aus dem Pflegedienst mit 22\% NSV ( $n=90 / 405)$. Im Zentrum der Chirurgie waren sowohl die Ärzte - [69,5\% $(\mathrm{n}=57 / 82)]$, als auch die Mitarbeiter aus dem Pflegedienst [31,4\% ( $n=32 / 102)]$ häufiger von NSV betroffen als die Mitarbeiter anderer Fachbereiche.

Das Risiko einer NSV war abhängig von der Art der Tätigkeit. Mitarbeiter aus den unterschiedlichen Fachdisziplinen wiesen unterschiedliche Unfallschwerpunkte auf. Die Art der invasiven Tätigkeit, bei der sich der Mitarbeiter verletzte, ist in - Abb. 3 dargestellt. Erwartungsgemäß stellte das Nähen in der Chirurgie das Hauptrisiko für eine NSV dar $(28,6 \%$; $n=96 / 336$ ), gefolgt von intraoperativen Schnittverletzungen $(12,5 \% ; n=42 / 336)$ sowie NSV bei der venösen Blutentnahme $(6 \% ; n=20 / 336)$. Auffällig war, dass in der Chirurgie insgesamt $45,2 \%(n=152 / 336)$ der NSV als „Sonstige Verletzungen“ klassifiziert wurden. Hierbei handelte es sich hauptsächlich um intraoperative Verletzungen durch Knochensplitter.

Nur 28,7\% der betroffen Mitarbeiter hatten alle NSV gemeldet. Innerhalb der einzelnen Berufsgruppen zeigten die Ärzte das schlechteste Meldeverhalten, nur 20,4\% der betroffenen Ärzte hatten alle NSV gemeldet, im Vergleich dazu hatten 40\% der Mitarbeiter des Pflegediensts alle stattgehabten NSV gemeldet. Chirurgen zeigten ein deutlich schlechteres Meldeverhalten (17,2\%) als chirurgisches Assistenzpersonal, welches in $52,5 \%$ der Fälle alle stattgehabten NSV gemeldet hatte (• Abb. 4).

In der Frankfurter Nadelstichstudie wurde ermittelt, welche NSV mit Hilfe von sicheren Produkten hätten vermieden werden können. Dabei wurde differenziert nach: höchstwahrscheinlich, vielleicht oder nicht vermeidbar (Einteilungskriterien der NSV s. Wicker et al. [21]). Durchschnittlich 36,4\% $(n=204 / 561)$ der NSV hätten durch die Verwendung von sicheren Produkten vermieden werden können, dabei zeigt sich ein signifikanter Unterschied innerhalb der einzelnen Fachdisziplinen (• Abb. 5):

- Pädiatrie $82,6 \%$

- Gynäkologie 81,4\%

- Anästhesie 53,7\%

- Dermatologie $41,7 \%$

- Chirurgie $14,6 \%$

\section{Diskussion}

Nadelstichverletzungen bedeuten für die Betroffenen ein nicht unerhebliches Infektionsrisiko bezüglich der über Blut übertragbaren Erreger HBV, HCV und HIV [9]. In diesen Kontext spielen Chirurgen bzw. D-Ärzte eine wichtige Rolle, da sie als D-Ärzte in der Regel die ersten Ansprechpartner für betroffene Beschäftigte darstellen und somit den weiteren Behandlungsprozess maßgeblich koordinieren sollten. In einer Studie des Instituts für Arbeitsmedizin der Freien Universität Berlin und der Humboldt-Univer- 
sität Berlin zeigten sich jedoch erhebliche Defizite hinsichtlich des Kenntnisstands der erstversorgenden Ärzte. Bemerkenswert in diesen Zusammenhang war beispielsweise, dass nur wenige D-Ärzte genaue Angaben über das korrekte Vorgehen nach einer HBV- bzw. HIV-Exposition machen konnten. Auch das Übertragungsrisiko von HBV (bis zu 30\% bei ungeimpften Personen) wurde eher unterschätzt, wohingegen das Risiko für eine Übertragung von HCV (etwa 1,5-3\%) bzw. HIV (etwa $0,3 \%$ ) eher überschätzt wurde. Es ergab sich somit eine gefährliche Kombination aus der Unterschätzung des Übertragungsrisikos für HBV und der Unkenntnis hinsichtlich der korrekten Behandlung nach HBV- bzw. HIVExposition [10].

Chirurgen haben aufgrund ihrer eigenen beruflichen Tätigkeit ein großes Risiko, eine NSV zu erleiden [19]. In der Frankfurter Nadelstichstudie waren die Mitarbeiter aus dem Zentrum für Chirurgie am häufigsten von NSV betroffen, pro Mitarbeiter und Jahr wurden 1,7 NSV angegeben.

In einer amerikanischen Studie war die Verletzungsrate bei Chirurgen in der Gruppe der Fachärzte mit 2,6 NSV/10o Vorgänge sowie mit 2,3 NSV/10o Vorgänge bei Assistenzärzten am höchsten [5]. In einer anderen amerikanischen Studie gaben 6-50\% der OP- und Kreißsaalbeschäftigten mindestens einen Blutkontakt pro Vorgang an, von denen wiederum 1-15\% zumindest eine NSV einschlossen [1].

Einen ausreichenden Hepatitis-B-Immunschutz gaben 95,1\% der von uns befragten Ärzte aus der Chirurgie an. Das Risiko für einen chirurgisch tätigen Arzt, eine HCV-Infektion durch eine berufliche Exposition zu erleiden, beträgt nach einer australischen Studie nach $35 \mathrm{Be}$ rufsjahren etwa 4,9\%. Insgesamt 3,8\% der Mitarbeiter wiesen eine HCV-RNA-positive PCR auf. Die geschätzte RNA-Positivität nach 15 Berufsjahren lag bei 1,6\% [17]. Deutliche geringere HCV-Transmissionsraten für Chirurgen fanden sich in einer schottischen Studie. Hier wurde aufgrund der ermittelten HCV-Prävalenz der Patienten von einer Transmission von $0,001-0,032 \% / J a h r(0,035-1,12 \%$ nach 35 Berufsjahren), mit einem maximalen
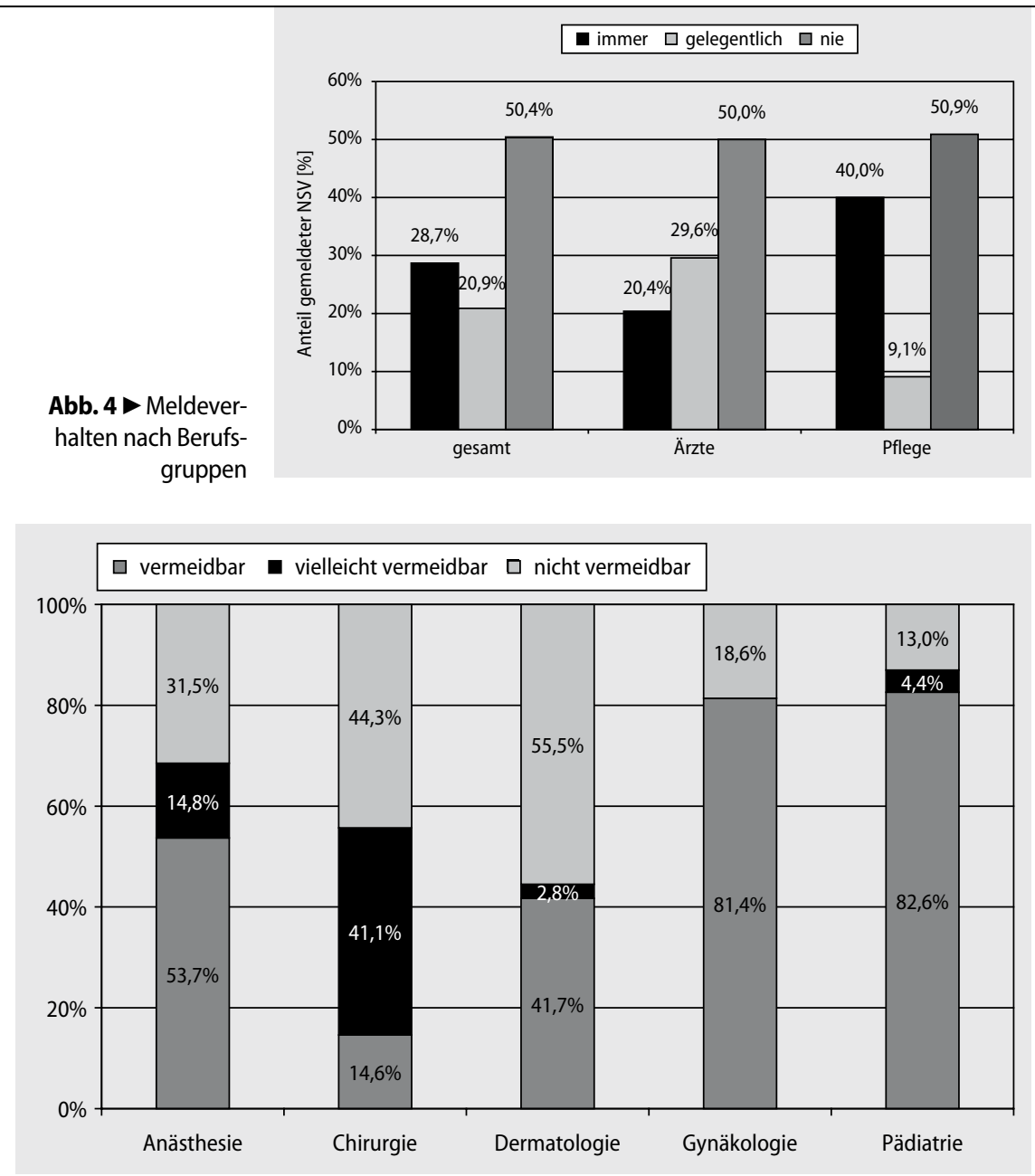

Abb. 5 A Vermeidbarkeit von NSV durch sichere Instrumente

1,4\%igen Risiko, im Rahmen des Berufslebens eine HCV-Infektion zu akquirieren, ausgegangen [14].

In einer französischen Studie gaben 60 bzw. $64 \%$ der befragten Mitarbeiter der Chirurgie an, nie zuvor auf HIV bzw. HCV getestet worden zu sein [13]. Dies ist gerade vor dem Hintergrund problematisch, dass einerseits bei einer frühzeitigen Diagnose und Therapie der akuten Hepatitis C eine Chronifizierung verhindert werden kann und andererseits ein infektiöser Chirurg ein Infektionsrisiko für die von ihm betreuten Patienten darstellt [18].

NSV verursachen alljährlich hohe Kosten in Deutschland. Laut Wittmann [22] belaufen sich die durchschnittlichen Kosten einer gemeldeten NSV auf knapp 500 EUR. Die Mehrkosten für eine komplette Substitution aller herkömmlichen Produkte für perkutane Eingriffe durch Sicherheitsprodukte dagegen betragen pro
Mitarbeiter und Jahr lediglich 63 EUR [22]. Die Gesamtkosten durch NSV belaufen sich in Deutschland auf etwa 50 Mio. EUR. Die Mehrkosten für die Einführung sicherer Instrumente liegen in einem ähnlichen Bereich, allerdings lassen sich durch deren Einsatz rund 85\% der NSV verhindern [23].

Sichere Instrumente, so das Ergebnis der Frankfurter Nadelstichstudie, reduzieren das Infektionsrisiko für Beschäftigte im Arbeits- und Gesundheitsdienst und verbessern den Schutz der Patienten. Zahlreiche Studien haben ihren Nutzen belegt $[6,11,12,15,21]$.

Zum jetzigen Zeitpunkt stehen jedoch noch nicht für alle invasiven Tätigkeiten adäquate Sicherheitsinstrumente zur Verfügung. So sind in der Chirurgie noch technische Verbesserungen der Instrumente erforderlich, um eine sichere und funktionelle Nutzung zu ermöglichen. 


\section{Fazit für die Praxis}

\section{Es ist dringend geboten, die Mitarbeiter im Gesundheitsdienst vor NSV und über blutübertragbaren Infektionen zu schüt- zen. Zahlreiche unabhängige Studien ha- ben den Nutzen von sicheren Instrumen- ten belegt. Die Einführung sicherer Pro- dukte sowie zielführende und angemes- sene Veränderungen des Arbeitsablaufs werden nachhaltige Effekte in der Prä- vention zeigen.}

\section{Korrespondenzadresse \\ Dr. S. Wicker}

Betriebsärztlicher Dienst, Klinikum, Johann-Wolfgang-Goethe-Universität Frankfurt, Theodor-Stern-Kai 7, 60590 Frankfurt am Main Sabine.Wicker@kgu.de

Interessenkonflikt. Der korrespondierende Autor gibt an, dass kein Interessenkonflikt besteht.

\section{Literatur}

1. Berry AJ, Greene ES (1992) The risk of needlestick injuries and needlestick-transmitted diseases in the practice of anaesthesiology. Anesthesiology 77: 1007-1021

2. DAIG, ÖAG, RKI (2003) HIV/AIDS-Postexpositionsprophylaxe. Deutsch-Östereichische Empfehlung zur postexpositionellen Prophylaxe der HIV-Infektion. Dtsch Med Wochenschr [Suppl 1] 128: 36-50

3. Hofmann F (2000) Arbeitsunfälle. In: Hofmann F (Hrsg) Betriebsarzt im Krankenhaus - Infektionsprophylaxe, Begehung und Ergonomie. ecomed, Landsberg, S 42-43

4. Hofmann F, Kralj N, Beie M (2002) Kanülenstichverletzungen im Gesundheitsdienst - Häufigkeit, Ursachen und Präventionsstrategien. Gesundheitswesen 64: 259-266

5. Lewis FR, Short LJ, Howard RJ et al. (1995) Epidemiology of injuries by needles and other sharp instruments. Minimizing sharp injuries in gynaecologic and obstetric operations. Surg Clin North Am 75: 1105-1121

6. Müller-Barthelmeh R, Buchholz L, Nübling M et al. (2006) Qualitätssicherung bei Nadelschutztechniken. Arbeitsmed Sozialmed Umweltmed 41: 210-217

7. NN (2003/2006) Technische Regeln für Biologische Arbeitsstoffe. Biologische Arbeitsstoffe im Gesundheitswesen und in der Wohlfahrtspflege (TRBA 250). BArbBL 11:53-73. Überarbeitete Version. 2006 BArbBI 7: 193

8. Prüss-Üstün A, Rapiti E, Hutin Y (2005) Estimation of the global burden of disease attributable to contaminated sharps injuries among health-care workers. Am J Ind Med 48: 482-490

9. Rabenau HF, Wicker S (2007) Gefährdungspotenzial durch Nadelstichverletzungen: Eine internationale Herausforderung. Krankenhaus-Hygiene Infektionsverh 3: 82-85

10. Samwer H, Sander M, Schäcke G (2006) Optimierung der Erstbehandlung beruflicher Nadelstichverletzungen. Zentralbl Arbeitsmed 56: 353-368
11. Sulsky SI, Birk T, Cohen LC et al. (2006) Effectiveness of measures to prevent needlestick injuries among employees in health professions. ENVIRON International Corporation, Health Science Institute, Baltimore

12. Suzuki R, Kimura S, Shintani Y et al. (2006) The efficacy of safety winged steel needles on needlestick injuries. Kansenshogaku Zasshi 80: 39-45

13. Tarantola A, Golliot F, L'Heriteau F et al. (2006) Assessment of preventive measures for accidental blood. Am J Infect Control 34: 376-382

14. Thorburn D, Roy K, Cameron SO (2003) Risk of hepatitis $C$ virus transmission from patients to surgeons: model based on an unlinked anonymous study of hepatitis $C$ virus prevalence in hospital patients in Glasgow. Gut 52: 1333-1338

15. Visser $L$ (2006) Toronto hospital reduces sharp injuries by $80 \%$, eliminates blood collected injuries. $A$ case study: Toronto East General Hospital pioneers healthcare worker safety. Healthc Q 1: 68-70

16. Wallis GC, Kim WY, Chaudhary BR et al. (2007) Perceptions of orthopaedic surgeons regarding hepatitis $C$ viral transmission: a questionnaire survey. Ann R Coll Surg Engl 89: 276-80

17. Watson KJR (2004) Surgeon, test (and heal) thyself: sharps injuries and hepatitis C risk. Med J Aust 181: 366-367

18. Wicker S (2006) Umgang mit infektiösen Beschäftigten - Aufgaben des Betriebsarztes. Krankenhaus-Hygiene Infektionsverh 28: 16-20

19. Wicker S, Rabenau HF (2007) Nadelstichverletzungen bei Mitarbeitern im Gesundheitswesen: Berufsrisiko oder vermeidbare Infektionsgefährdung? Krankenhaus-Hygiene Infektionsverh 3: 8690

20. Wicker S, Allwinn R, Gottschalk R et al. (2007) Häufigkeit von Nadelstichverletzungen in einem deutschen Universitätsklinikum: Ein Vergleich zweier unabhängiger Datenerhebungen. Zentralbl Arbeitsmed 57: 42-49

21. Wicker S, Jung J, Allwinn R et al. (2007) Prevalence and prevention of needlestick injuries among health care workers in a German university hospital. Int Arch Occup Environ Health (in press) DOI 10.1007/s00420-007-0219-7

22. Wittmann A (2006) Kosten von Nadelstichverletzungen und wirtschaftlicher Nutzen neuer Sicherheitsprodukte. Prakt Arbeitsmed 5: 40-541

23. Wittmann A, Zylka-Menhorn V (2007) Verletzungssichere Instrumente für Kliniken und Praxen obligatorisch. Dtsch Arztebl 104: C 624-626 